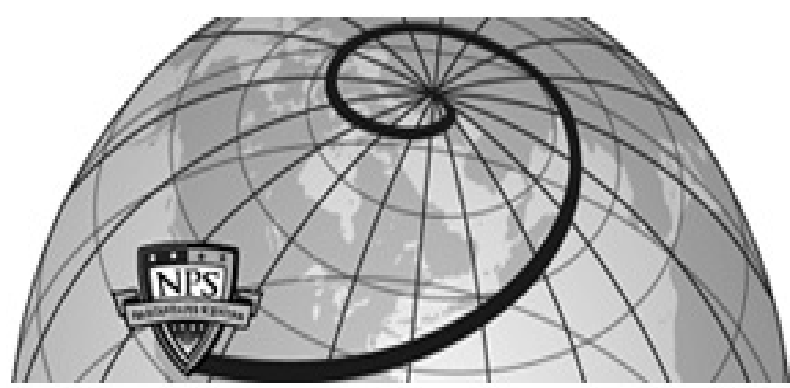

Calhoun: The NPS Institutional Archive DSpace Repository

\title{
On Discrete-Time Optimality Conditions for Pseudospectral Methods
}

Fahroo, Fariba; Ross, I. Michael

The American Institute of Aeronautics and Astronautics (AIAA)

https://hdl.handle.net/10945/29663

This publication is a work of the U.S. Government as defined in Title 17, United States Code, Section 101. Copyright protection is not available for this work in the United States.

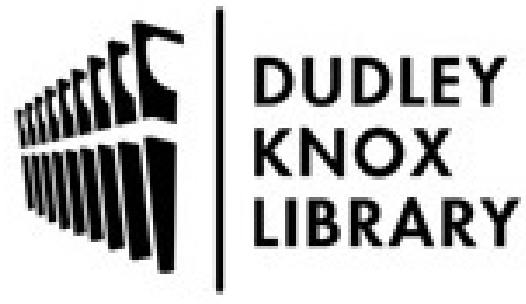

http://www.nps.edu/library
Calhoun is the Naval Postgraduate School's public access digital repository for research materials and institutional publications created by the NPS community. Calhoun is named for Professor of Mathematics Guy K. Calhoun, NPS's first appointed -- and published -- scholarly author.

\section{Dudley Knox Library / Naval Postgraduate School} 411 Dyer Road / 1 University Circle Monterey, California USA 93943 


\title{
On Discrete-Time Optimality Conditions for Pseudospectral Methods
}

\author{
Fariba Fahroo* I. Michael Ross ${ }^{\dagger}$
}

\begin{abstract}
One of the most efficient families of techniques for solving space trajectory optimization problems are pseudospectral (PS) methods. Among the rich variety of PS methods, the class of Legendre PS methods are most thoroughly studied for optimal control and trajectory optimization applications. In particular, the Legendre-Gauss-Lobatto PS method is widely used for boundary-value type problems while the Legendre-Gauss-Radau PS method was recently proposed for solving infinite-horizon optimal control problems as a means to manage conditions at infinity. Both methods satisfy the Covector Mapping Principle, the mathematical principle associated with the consistency of approximations that allows one to generate dual maps (such as Hamiltonians, adjoints etc) without resorting to solving difficult two-point boundary-value problems. In this paper we prove that a combination of weighted interpolants, their duals, and a proper definition of orthogonality allows us to formulate a generalized Covector Mapping Theorem that applies to all such PS methods. The consequences of this theorem are that it clarifies the connections between theory and computation, the impact of these connections on solving trajectory optimization problems, and the selection of the correct PS method for solving problems quickly and efficiently. A classical benchmark continuous-thrust orbit transfer problem is used to illustrate the concepts.
\end{abstract}

\section{Introduction}

In recent years, a vast number of space trajectory optimization problems have been solved by pseudospectral (PS) methods; examples include the design of Earth-Mars return trajectories, ${ }^{1}$ lunar landing, ${ }^{2}$ librationpoint stationkeeping, ${ }^{3}$ solar-sail cyclers ${ }^{4}$ asteroid sample return mission design, ${ }^{5,6}$ inert and electrodynamic tether control,,${ }^{7,8}$ libration-point formation design and control, ${ }^{9}$ aerocapture, ${ }^{10}$ ascent,${ }^{5,11,12}$ entry guidance ${ }^{13-16}$ almost-periodic formations,${ }^{9,17,18}$ formation reconfiguration problems, ${ }^{19}$ low-thrust trajectory optimization, ${ }^{20-22}$ high-thrust optimization problems ${ }^{23}$ and many more; see for example, Refs. [18] and [24] for a review of the problems, methods and issues. We briefly note that PS methods are not limited to space trajectory optimization problems but are applicable to optimal control and dynamic optimization problems in general. For example, PS methods have been successfully applied for singularity-free maneuvering of controlmoment gyros ${ }^{25}$ (CMGs), CMG momentum-dumping, ${ }^{26}$ magnetic attitude control problems, ${ }^{27,28}$ unmanned aerial vehicle endurance problems, ${ }^{29}$ crane-and-pulley problems, ${ }^{30}$ robotic arm control, ${ }^{31}$ nonlinear observer ${ }^{32}$ etc. As a result of their versatility, PS methods are now available in NASA's OTIS software package. ${ }^{33}$ Details of these plans are described in http://trajectory.grc.nasa.gov/projects/lowthrust.shtml. The essential idea of making PS methods available through OTIS is that users who are familiar with the OTIS interface can now make avail of PS methods simply by setting a few options.

The most widely used PS method is the Legendre PS method ${ }^{22,34,35}$ that is based on the Legendre-GaussLobatto (LGL) node points. This is simply because the LGL/PS method is a complete method; that is, it makes no distinction between the so-called direct and indirect methods. ${ }^{36,37}$ This implies that a practitioner may treat the outcome of the LGL/PS method in terms of a rigorous application of the Pontryagin Minimum Principle and accept or reject solutions based on the optimality conditions. This comfort of guarantees is enunciated as the Covector Mapping Principle ${ }^{38-40,42}$ (CMP) which provides the foundations for generating

*Associate Professor, Department of Applied Mathematics, Naval Postgraduate School, Monterey, CA $93943 . \quad$ Email: ffahroo@nps.edu. Associate Fellow, AIAA

${ }^{\dagger}$ Professor, Department of Mechanical \& Astronautical Engineering, Code ME/Ro, Naval Postgraduate School, Monterey, CA 93943; imross@nps.edu. Associate Fellow, AIAA. 
explicit Covector Mapping Theorems. ${ }^{34,35}$ The proof of this theorem utilizes specific quadrature formulas that have thus far shown to be valid only for the LGL/PS method, and hence the popularity of this approach.

Space trajectory optimization problems are fundamentally boundary-value problems (BVPs). In certain instances, particularly in the broader class of optimal control problems, the final-time conditions may be free. Although the LGL/PS method continues to hold in this instance as well, the Legendre-Gauss-Radau (LGR) PS method ${ }^{43}$ provides an elegant approach to handle potential singularity problems that arise as a result of a time-domain transformation. Recent studies show a number of other advantages of the LGR/PS method, particularly for real-time applications. ${ }^{44}$ This generates a natural question: does the LGR/PS method satisfy the CMP? An apparently simple way to investigate this issue is to derive an explicit covector map ${ }^{24}$ in a manner similar to that of the LGL/PS approach. While conceptually simple, this task is not altogether straightforward as a key lemma related to an integration-by-parts formula ${ }^{35}$ is not readily available for non-LGL methods. This crucial formula identifies the correct finite-dimensional inner-product space that is necessary for the construction of the discretized 1-form that defines the sequences of discretized Lagrangians that converge to the continuous-time Lagrangian. ${ }^{45}$ In this paper, we identify this pre-Hilbert space by investigating the problem at the level of first principles. The concept of a pre-Hilbert space is crucial for proofs of convergence theorems ${ }^{46-48}$ that rely on the separability of the infinite-dimensional Hilbert spaces used to construct highly-accurate solutions to practical trajectory optimization problems.

In order to convey our ideas to a broad audience, we provide in this paper the foundations of PS methods for optimal control in a manner that is applicable to all Legendre-based PS methods: LGL, LGR and Legendre-Gauss (LG). The key idea introduced in this paper is the notion of weighed interpolants, their duals, and their direct effect on the generation of the correct pre-Hilbert space where the computed solutions lie. This concept demonstrates that the LGL/PS is indeed the correct Legendre PS method for solving non-homogenous BVPs and hence all space trajectory optimization problems. Furthermore, our concept illustrates why the LGR/PS method is indeed better than the LGL/PS method for stabilizing control systems. Finally, we show by direct numerical computation that when the LGR/PS and LG/PS methods are artificially forced to solve BVP-type problems, the results are computationally indistinguishable from the LGL/PS method. Our theory explains this phenomenon thus suggesting that the weighted-interpolant perspective is indeed the proper perspective for all PS methods for optimal control. A complete description of this new approach to PS methods is beyond the scope of this paper, and hence the ideas proposed here must be construed as illustrative. Nonetheless, some remarks on the general principles are provided at the end of this paper.

\section{A Distilled Trajectory Optimization Problem}

For simplicity in exposition, we will consider the following scalar Bolza problem, with the understanding that our discretization methods can be easily extended to higher-dimensional cases. For the same reason we will ignore path constraints as these can also be easily incorporated into our framework by replacing the control Hamiltonian by the Lagrangian of the Hamiltonian. ${ }^{35}$ In simplifying the bookkeeping, consider then the problem of finding the optimal state-control function pair $t \mapsto(x, u) \in \mathbb{R} \times \mathbb{R}$ that solves the following problem (Problem B):

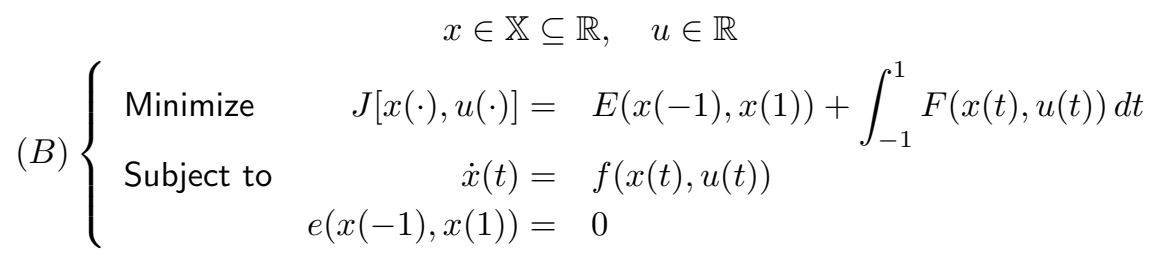

where $\mathbb{X}$ is an open set in $\mathbb{R}$ and the problem data (i.e. the endpoint cost function, $E$, the running cost function, $F$, the vector field, $f$ and the endpoint constraint function, $e$ ) are assumed to be at least $C^{1}$-smooth. An application of Pontryagin's Minimum Principle (PMP) results in a two-point BVP that we denote as Problem $B^{\lambda}$. This "dualization" is achieved through the construction of the control Hamiltonian, $H$, and endpoint Lagrangian, $\bar{E}$, defined as

$$
\begin{aligned}
H(\lambda, x, u) & =F(x, u)+\lambda f(x, u) \\
\bar{E}(\nu, x(-1), x(1)) & =E(x(-1), x(1))+\nu e(x(-1), x(1))
\end{aligned}
$$


where $\lambda$ is the adjoint covector (or costate) and $\nu$ is the endpoint covector. Problem $B^{\lambda}$ is now posed as follows:

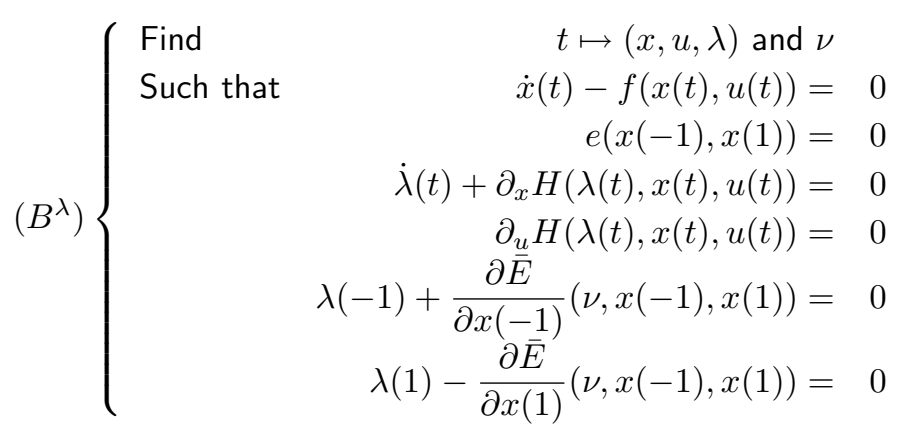

In using the PMP to solve a trajectory optimization problem, we must solve Problem $B^{\lambda}$ which is clearly a problem of finding the zeros of a map in an appropriate function space. ${ }^{38}$ This is the so-called indirect method. From a first-principles perspective, ${ }^{49}$ the PMP is used as follows: For every optimal solution to Problem $B$, there exist a costate function, $t \mapsto \lambda$ and an endpoint covector, $\nu$, that satisfy the conditions set forth by Problem $B^{\lambda}$. Thus, every suspect optimal solution to Problem $B$ must also be a solution to Problem $B^{\lambda}$ under appropriate technical conditions. The LGL/PS method ensures a satisfaction of this condition through its Covector Mapping Theorem. ${ }^{34,35}$ A generalized version of this theorem with respect to PS methods is the main contribution of this paper and is developed in the following sections. A generalized version of this theorem with respect to the stability and convergence of the approximation is described in Ref. [48].

\section{A New Perspective on Pseudospectral Methods}

The class of Legendre PS methods are a subset of a larger class of spectral methods which use orthogonal basis functions in global expansions similar to Fourier and Sinc series expansions. ${ }^{50}$ PS methods are based on approximating the unknown functions by weighted interpolants, ${ }^{51}$ where the interpolating points can be any of the three major classes of Gaussian quadrature points called nodes: Gauss points, Radau points and Lobatto points. Note that Radau and Lobatto points are also Gaussian quadrature points; this is why specific PS methods are identified by adjectives that describe the specific choice of nodes. All of these nodes are zeros of the orthogonal polynomials or their derivatives such as Legendre and Chebyshev or more generally the Jacobi polynomials. Thus, a very large family of PS methods can be generated to solve optimal control problems. This notion is similar to Runge-Kutta (RK) methods. For example, Euler, trapezoid, HermiteSimpson and many other methods are equivalently some form of an RK method with appropriately chosen coefficients. $^{37}$ Just as not all RK methods are legitimate, not all PS methods are legitimate. This is particularly true in solving optimal control problems as has been noted by Hager. ${ }^{38}$ For example, an RK method that is legitimate for propagating an ODE may fail gloriously when applied to an optimal control problem. In other words, it is imperative to carefully select an appropriate method to solve optimal control problems.

In our previous papers, we presented methods based on Lobatto points of Chebyshev ${ }^{52}$ and Legendre polynomials. ${ }^{35}$ In the absence of special information (such as special boundary conditions), it is customary to select Chebyshev PS methods in solving PDE problems as they are simple and effective. For optimal control of ODEs, the LGL/PS method is preferable as it maintains the consistency of dual approximations similar to the Hager class of RK methods. Recently, we proposed the usage of Radau point ${ }^{43,44}$ as a means to manage a singularity problem that arises in solving infinite-horizon control problems. The goal of this paper is to present a more unified view of these methods where we can show the similarities and differences of the methods based on the choice of these different nodes and show the effect of these nodes on the discretization of both the primal and dual problems.

\section{A. Weighted Interpolants and Differentiation Matrices}

We begin with the basic definition of PS methods and the different ingredients required in defining these methods for various nodes. Following Weideman, ${ }^{51}$ we select PS methods based on weighted interpolants of 
the form,

$$
y(t) \approx y^{N}(t)=\sum_{j=0}^{N} \frac{W(t)}{W\left(t_{j}\right)} \phi_{j}(t) y_{j}, a \leq t \leq b
$$

where $y(t)$ is an arbitrary function. Here the nodes $t_{j}, j=0, \ldots, N$ are a set of distinct interpolation nodes (defined below) in the interval $[a, b]$, the weight function $W(t)$ is a positive function on the interval, $y_{j}$ is a shorthand notation for $y^{N}\left(t_{j}\right)$, and $\phi_{j}(t)$ is the $N t h$ - order Lagrange interpolating polynomial that satisfies the relationship $\phi_{j}\left(t_{k}\right)=\delta_{j k}$. An expression for the Lagrange polynomial can be written as ${ }^{50}$

$$
\phi_{j}(t)=\frac{g_{N}(t)}{g_{N}^{\prime}\left(t_{j}\right)\left(t-t_{j}\right)}, g_{N}(t)=\prod_{j=0}^{N}\left(t-t_{j}\right) .
$$

One important tenant of polynomial approximation of functions is that differentiation of the approximated functions can be performed by differentiation of the interpolating polynomial,

$$
\frac{d y(t)}{d t}=\sum_{j=0}^{N} \frac{y_{j}}{W\left(t_{j}\right)}\left[W^{\prime}(t) \phi_{j}(t)+W(t) \phi_{j}^{\prime}\right]
$$

Since only the values of the derivative at the nodes $t_{i}$ are required for PS methods, then we have,

$$
\left.\frac{d y(t)}{d t}\right|_{t_{i}}=\sum_{j=0}^{N} \frac{y_{j}}{W\left(t_{j}\right)}\left[W^{\prime}\left(t_{i}\right) \delta_{i j}+W\left(t_{i}\right) D_{i j}\right]=\sum_{j=0}^{N} D_{i j}[W] y_{j}
$$

where we use $D_{i j}[W]$ as a shorthand notation for the $W$-weighted differentiation matrix,

$$
D_{i j}[W]=\frac{\left[W^{\prime}\left(t_{i}\right) \delta_{i j}+W\left(t_{i}\right) D_{i j}\right]}{W\left(t_{j}\right)}
$$

and $D_{i j}$ is usual unweighted differentiation matrix given by,

$$
D_{i j}=\left.\frac{d \phi_{j}(t)}{d t}\right|_{t=t_{i}}
$$

Thus, when $W(t)=1$, we have

$$
D_{i j}[1]=D_{i j}
$$

From Eq. (4), the unweighted differentiation matrix, $D_{i j}=\phi_{j}^{\prime}\left(t_{i}\right)$, has the form,

$$
D_{i j}= \begin{cases}\frac{g_{N}^{\prime}\left(t_{i}\right)}{g_{N}^{\prime}\left(t_{j}\right)} \frac{1}{\left(t_{i}-t_{j}\right)}, & i \neq j \\ \frac{g_{N}^{\prime \prime}\left(t_{i}\right)}{2 g_{N}^{\prime}\left(t_{i}\right)}, & i=j\end{cases}
$$

The above equations are the general representations of the derivative of the Lagrange polynomials evaluated at the interpolation nodes.

\section{B. Legendre Nodes}

By definition, PS methods use Gaussian quadrature points which lie in the interval $[-1,1]$ in order to obtain the best error estimates for integration, differentiation and interpolation of functions. ${ }^{53}$ In most problems the physical problem is posed on some interval $[a, b]$ which can then be easily transformed to the computational domain $[-1,1]$ via a linear transformation. The Gauss quadrature points are zeros that are interior to the interval $[-1,1]$. The Gauss-Radau zeros include one of the end points of the interval, usually the left-end point at $t=-1$. The Gauss-Lobatto points include both endpoints of the interval at $t=-1$, and $t=1$. These 
quadrature nodes are related to the zeros of the $Q$ th-order Jacobi polynomials $P_{Q}^{\alpha, \beta}$ which are orthogonal on the interval $[-1,1]$ with respect to the inner product, ${ }^{53,54}$

$$
\int_{-1}^{1}(1-t)^{\alpha}(1+t)^{\beta} P_{Q}^{\alpha, \beta}(t) P_{Q^{\prime}}^{\alpha, \beta}(t) d t
$$

Note that the Legendre polynomials are a special case of Jacobi polynomials which correspond to $\alpha=\beta=0$. Let $t_{i, Q}^{\alpha, \beta}$ be the $Q$ zeros of the $Q t h$-order Jacobi polynomial $P_{Q}^{\alpha, \beta}$ such that

$$
P_{Q}^{\alpha, \beta}\left(t_{i, Q}^{\alpha, \beta}\right)=0, \quad i=0, \ldots, Q-1
$$

Then, we can define zeros and weights that approximate the following integral

$$
\int_{-1}^{1} u(t) d t=\sum_{i=0}^{N} w_{i} u\left(t_{i}\right)+R(u)
$$

and find expressions for the unweighted differentiation matrices for these nodes as follows: ${ }^{4}$

1. Legendre-Gauss ( $L G$ )

$$
\begin{gathered}
t_{i}=t_{i, N+1}^{0,0}, \quad i=0, \ldots, N \\
w_{i}^{0,0}=\frac{2}{1-\left(t_{i}\right)^{2}}\left[\frac{d}{d t}\left(\left.L_{N+1}(t)\right|_{t=t_{i}}\right]^{-2}, \quad i=0, \ldots, N,\right. \\
R(u)=0 \text { if } u(t) \in \mathcal{P}^{2 N+1}([-1,1]) . \\
D_{i j}= \begin{cases}\frac{L_{N+1}^{\prime}\left(t_{i}\right)}{L_{N+1}^{\prime}\left(t_{j}\right)\left(t_{i}-t_{j}\right)}, & i \neq j, 0 \leq i, j \leq N \\
\frac{t_{i}}{1-t_{i}{ }^{2}}, & i=j\end{cases}
\end{gathered}
$$

2. Legendre-Gauss-Radau (LGR)

$$
\begin{aligned}
& t_{i}=\left\{\begin{aligned}
-1, & i & =0, \\
t_{i-1, N}^{0,1}, & i & =0, \ldots, N
\end{aligned}\right. \\
& w_{i}^{0,0}=\frac{1-t_{i}}{(N+1)^{2}\left[L_{N}\left(t_{i}\right)\right]^{2}}, i=0, \ldots, N \text {, } \\
& R(u)=0 \text { if } u(t) \in \mathcal{P}^{2 N}([-1,1]) . \\
& D_{i j}= \begin{cases}\frac{-N(N+2)}{4}, & i=j=0, \\
\frac{L_{N}\left(t_{i}\right)}{L_{N}\left(t_{j}\right)} \frac{1-t_{j}}{1-t_{i}} \frac{1}{\left(t_{i}-t_{j}\right)}, & i \neq j, 0 \leq i, j \leq N \\
\frac{1}{2\left(1-t_{i}\right)}, & 1 \leq i=j \leq N\end{cases}
\end{aligned}
$$

3. Legendre-Gauss-Lobatto ( $L G L)$

$$
\begin{gathered}
t_{i}= \begin{cases}-1, & i=0, \\
t_{i-1, N-1}^{1,1}, & i=1, \ldots, N-1 \\
1 & i=1\end{cases} \\
w_{i}^{0,0}=\frac{2}{N(N+1)\left[L_{N}\left(t_{i}\right)\right]^{2}}, i=0, \ldots, N, \\
R(u)=0 \text { if } u(t) \in \mathcal{P}^{2 N-1}([-1,1]) .
\end{gathered}
$$




$$
D_{i j}= \begin{cases}\frac{-N(N+1)}{4}, & i=j=0, \\ \frac{L_{N}\left(t_{i}\right)}{L_{N}\left(t_{j}\right)} \frac{1}{\left(t_{i}-t_{j}\right)}, & i \neq j, 0 \leq i, j \leq N \\ 0, & 1 \leq i=j \leq N-1 \\ \frac{N(N+1)}{4}, & i=j=N,\end{cases}
$$

where $\mathcal{P}^{N}$ is the set of all algebraic polynomials of degree at most $N$.

\section{Pre-Hilbert Spaces}

Given the definitions of the quadrature nodes and weights above, we can proceed with the definitions of the appropriate discrete inner-product spaces associated with the above nodes and weights. Let $[-1,1] \mapsto$ $\{y(t), z(t)\}$ be real-valued functions in $L^{2}([-1,1], \mathbb{R})$. The standard inner product in $L^{2}$ is given by

$$
\langle y, z\rangle_{L^{2}}:=\int_{-1}^{+1} y(t) z(t) d t
$$

For any given $m \in \mathbb{N}$, and $\left\{w_{j}>0, j=0,1, \ldots m\right\}$, define the weighted discrete inner product in $L^{2}$ to be

$$
\langle y, z\rangle_{m, w}:=\sum_{j=0}^{m} y\left(t_{j}\right) w_{j} z\left(t_{j}\right)
$$

For the various Gauss quadrature nodes and the standard inner-product in $L^{2}$, we have the following result: ${ }^{50}$

Lemma 1 For all $p q \in \mathcal{P}^{2 N+\zeta,}$

$$
\langle p, q\rangle_{L^{2}}=\langle p, q\rangle_{N, w}
$$

where $\zeta=1$ for Legendre-Gauss $(L G), \zeta=0$ Legendre-Gauss-Radau (LGR) and $\zeta=-1$ for Legendre-GaussLobatto (LGL) integration and weights.

For any weight function, $W(t)$, the weighted inner product in $L^{2}$ is given by

$$
\langle y, z\rangle_{L_{W}^{2}}:=\int_{-1}^{+1} y(t) W(t) z(t) d t
$$

Let $W(t)$ be any one of the following weight functions,

$$
W(t)= \begin{cases}W_{l g l}(t) & \Rightarrow W(t)=1 \\ W_{l g r}(t) & \Rightarrow W(t)=1-t \\ W_{l g}(t) & \Rightarrow W(t)=1-t^{2}\end{cases}
$$

From Lemma 1, we have the following unified result:

Lemma 2 For all $p q \in \mathcal{P}^{2 N-1}$,

$$
\langle p, q\rangle_{L_{W}^{2}}=\langle p, q\rangle_{N, w}
$$

Lemma 2 implies that $W_{l g l}$ is the only weight function that is dual to itself in the standard inner product space and unweighted interpolation.

Lemma 3 For the three Legendre quadrature nodes, $L G, L G R$ and $L G L$, the following relationships hold: For $i \neq j$ we have,

$$
D_{i j}=-\frac{W\left(t_{j}\right)}{W\left(t_{i}\right)} \frac{w_{j}}{w_{i}} D_{j i}
$$

Hence, we can write,

$$
D_{i j}[W]=-\frac{w_{j}}{w_{i}} D_{j i}, i \neq j
$$


and for $i=j$ the following relationships hold:

$$
\begin{aligned}
\text { For } L G \text { Nodes } & \rightarrow \quad D_{i i}\left[W_{l g}\right]=-D_{i i}, i=0, \cdots, N \\
\text { For LGR Nodes } & \rightarrow\left\{\begin{array}{l}
D_{i i}\left[W_{l g r}\right]=-D_{i i} i=1, \cdots, N \\
D_{00}\left[W_{l g r}\right]=-D_{00}-\frac{1}{w_{0}}
\end{array}\right. \\
\text { For LGL Nodes } & \rightarrow\left\{\begin{array}{l}
D_{i i}\left[W_{l g l}\right]=D_{i i}=0, i=1, \cdots, N-1 \\
D_{00}\left[W_{l g l}\right]=-D_{00}-1 / w_{0} \\
D_{N N}\left[W_{l g l}\right]=-D_{N N}+1 / w_{N}
\end{array}\right.
\end{aligned}
$$

\section{Pseudospectral Methods for Trajectory Optimization}

We can now define LG, LGR and LGL pseudospectral methods based on the choice of interpolation nodes. For approximating the state and costate variables we use the expansions,

$$
x^{N}(t)=\sum_{j=0}^{N} \frac{W(t)}{W\left(t_{j}\right)} x_{j} \phi_{j}(t), \quad \lambda^{N}(t)=\sum_{j=0}^{N} \frac{W^{*}(t)}{W^{*}\left(t_{j}\right)} \lambda_{j} \phi_{j}(t)
$$

where $W$ and $W^{*}$ are an appropriate choice of weight functions. Lemma 2 suggests that if we take $W(t)=1$ and LGL nodes, then we must take $W^{*}(t)=1$ as well. This is the standard theory. ${ }^{35}$ It is clear that the use of Lemma 2 in the integration-by-parts argument proposed in Refs. [21,35], generalizes the standard theory by suggesting that if we take $W(t)=1$ and LGR nodes, then $W^{*}(t)=1-t$; likewise, for $W(t)=1$ and LG nodes, $W^{*}(t)=1-t^{2}$. Similarly, if we take $W(t)=1-t$ for LGR nodes, then $W^{*}(t)=1$ etc.; thus, $\left(W^{*}(t)\right)^{*}=W(t)$.

For approximating the control variables, we take

$$
u^{N}(t)=\sum_{j=0}^{N} u_{j} \psi_{j}(t)
$$

where $\psi_{j}(t)$ is any interpolant; see Refs. [46,48] for a justification of this principle. Based on these preliminary ideas, we formulate PS methods for trajectory optimization as follows: We set $W(t) \equiv 1$. for the state variables for all Legendre PS methods. This automatically defines appropriate weight functions for costate interpolation. Alternatively, we may set $W(t)$ according to the appropriate class of weighted interpolations in which case we would arrive at the corresponding duals. Applying the quadrature rules for approximating the integral terms and using the appropriate form of the derivative matrix according to the choice of the nodes, we have the following expressions for Problem $B^{N}$ which is the discretized form of Problem $B$ :

$$
\left(B^{N}\right)\left\{\begin{aligned}
X \in \mathbb{R}^{N_{n}}, \quad u \in \mathbb{R}^{N_{n}} & \\
\text { Minimize } & J^{N}[X, U]=\sum_{j=0}^{N} w_{j} F\left(x_{j}, u_{j}\right) \\
\text { Subject to } \quad \sum_{j=0}^{N} D_{i j} x_{j}-f\left(x_{i}, u_{i}\right) & =0 \quad i=0,1, \ldots, N \\
e\left(x_{0}, x_{N}\right) & =0
\end{aligned}\right.
$$

\section{A. Selection of a Proper Choice of PS Methods}

Lemma 2 and the imposition of the endpoint constraints as implied in Problem $B^{N}$ suggest the proper choice of an appropriate PS method. In many optimal control problems both endpoint conditions are specified; that is, conditions at the initial and final time. Then, according to Lemma 2 and the formulation of Problem $B^{N}$, we must choose the LGL/PS method. In optimal control problems that are based on stabilizing a control system, the initial conditions are specified but the final conditions are homogeneous. This suggests that the LGR/PS method may be used as $e(x(-1), x(1))$ is independent of $x(1)$. In principle an LGL/PS method may 
also be used with the final conditions set to zero. This concept would be consistent with the LGR/PS method provided the horizon was finite. For infinite horizon problems, this generates a singularity problem indicating that the LGR/PS method does indeed form the proper choice of a PS method for infinite horizon problems, and not merely a better method. For finite-horizon problems with both endpoints specified, the LGR/PS method is inappropriate as it suggests that the final time condition be artificially imposed at $x_{N}$, a point interior to the interval $[-1,1]$. Nonetheless, one can, in principle, construct such a PS method. Although dubious from a theoretical standpoint, numerical experiments suggest that its performance is numerically indistinguishable from the LGL/PS method for sufficiently large $N$. From a theoretical perspective, this can be justified in the sense that as $N \rightarrow \infty$ all three methods converge; see Ref. [46] for a rigorous proof of this point. Since convergence is at a spectral rate, numerical differences are not distinguishable for even low $N$. Given these observations, it is clear that the LGL/PS method constitutes a simpler and theoretically justified PS method for finite-horizon problems and unjustified for infinite-horizon problems whereas the LGR/PS method is an appropriate PS method for infinite-horizon problems. By the same token, the LG/PS method constitutes an appropriate PS method for optimal control problems with homogenous boundary conditions. For such problems the LGR and LGL pseudospectral methods may also be used with complete theoretical justification as implied by Lemma 2. The performance differences would be minor or indistinguishable due to the spectral convergence properties of all PS methods. On the other hand, if the LG/PS method is artificially used for non-homogenous boundary conditions by imposing them at the interior points of the interval $[-1,1]$, the same conclusions associated with the LGR/PS method hold with an additional dubiousness of conditions at the initial time.

\section{B. Discretization of the Dualized Problem $\left(B^{\lambda N}\right)$}

The costate derivative is approximated as,

$$
\left.\frac{d \lambda^{N}(t)}{d t}\right|_{t_{i}}=\sum_{j=0}^{N} D_{i j}^{*} \lambda_{j}
$$

with

$$
D_{i j}^{*}=D_{i j}[W]=\frac{\left[W^{\prime}\left(t_{i}\right) \delta_{i j}+W\left(t_{i}\right) D_{i j}\right]}{W\left(t_{j}\right)}
$$

Thus Problem $B^{\lambda N}$ can be constructed as,

$$
\left(B^{\lambda N}\right)\left\{\begin{array}{rlr}
\sum_{j=0}^{N} D_{i j} x_{j}-f\left(x_{i}, u_{i}\right) & =0 & i=0,1, \ldots, N \\
e\left(x_{0}, x_{N}\right) & =0 & \\
\sum_{j=0}^{N} D_{i j}^{*} \lambda_{j}+\partial_{x_{i}} H\left(\lambda_{i}, x_{i}, u_{i}\right) & =0 & i=0,1, \ldots, N \\
\partial_{u_{i}} H\left(\lambda_{i}, x_{i}, u_{i}\right) & =0 & i=0,1, \ldots, N \\
\lambda_{0}+\frac{\partial \bar{E}}{\partial x_{0}}\left(\nu, x_{0}, x_{N}\right) & =0 & \\
\lambda_{N}-\frac{\partial \bar{E}}{\partial x_{N}}\left(\nu, x_{0}, x_{N}\right) & =0 &
\end{array}\right.
$$

\section{A Generalized Covector Mapping Theorem}

Based on the preceding discussions it is now abundantly clear that the correct inner product space for all three discretizations is given by $\mathbb{R}_{w}^{N_{n}}$, the finite-dimensional Hilbert space $\mathbb{R}^{N_{n}}$ equipped with the weighted inner product,

$$
\langle a, b\rangle_{\mathbb{R}_{w}^{N_{n}}}:=\sum_{i=0}^{N} a_{i} w_{i} b_{i} \quad a, b \in \mathbb{R}^{N_{n}}
$$


Thus, the Lagrangian in $\mathbb{R}_{w}^{N_{n}}$ is given by, ${ }^{45}$

$$
\begin{aligned}
\overline{J^{N}}[\widetilde{\lambda}, \widetilde{\nu}, x, u] & =\sum_{j=0}^{N} w_{j} F\left(x_{j}, u_{j}\right)+\sum_{i=0}^{N} w_{i} \widetilde{\lambda}_{i} f\left(x_{i}, u_{i}\right)-\sum_{i=0}^{N} w_{i} \widetilde{\lambda}_{i} \sum_{j=0}^{N} D_{i j} x_{j}+\widetilde{\nu} e\left(x_{0}, x_{N}\right) \\
& =\sum_{i=0}^{N} w_{i} H\left(\widetilde{\lambda}_{i}, x_{i}, u_{i}\right)-\sum_{i=0}^{N} w_{i} \widetilde{\lambda}_{i} \sum_{j=0}^{N} D_{i j} x_{j}+\widetilde{\nu} e\left(x_{0}, x_{N}\right)
\end{aligned}
$$

where $\widetilde{\lambda}$ and $\widetilde{\nu}$ are the Karush-Kuhn-Tucker (KKT) multipliers in $\mathbb{R}_{w}^{N_{n}}$. From the KKT theorem we have,

$$
\begin{aligned}
\partial_{x_{k}} \overline{J^{N}}[\widetilde{\lambda}, \widetilde{\nu}, x, u] & =w_{k} \partial_{x_{k}} H\left(\widetilde{\lambda}_{k}, x_{k}, u_{k}\right)-\sum_{i=0}^{N} w_{i} \widetilde{\lambda}_{i} \sum_{j=0}^{N} D_{i j} \delta_{j k} \quad k=1, \ldots, N-1 \\
& =w_{k} \partial_{x_{k}} H\left(\widetilde{\lambda}_{k}, x_{k}, u_{k}\right)-\sum_{i=0}^{N} w_{i} \widetilde{\lambda}_{i} D_{i k}
\end{aligned}
$$

From Lemma 3 we have,

$$
D_{k i}^{*}:=-\frac{w_{i}}{w_{k}} D_{i k} \quad \text { for } k=1, \ldots, N-1, i=0, \ldots, N
$$

Hence,

$$
\partial_{x_{k}} \overline{J^{N}}[\widetilde{\lambda}, \widetilde{\nu}, X, U]=w_{k}\left(\partial_{x_{k}} H\left(\widetilde{\lambda}_{k}, x_{k}, u_{k}\right)+\sum_{i=0}^{N} D_{k i}^{*} \widetilde{\lambda}_{i}\right)=0
$$

Similarly,

$$
\begin{aligned}
\partial_{x_{k}} \overline{J^{N}}[\widetilde{\lambda}, \widetilde{\nu}, X, U] & =w_{k} \partial_{x_{k}} H\left(\widetilde{\lambda}_{k}, x_{k}, u_{k}\right)-\sum_{i=0}^{N} w_{i} \widetilde{\lambda}_{i} \sum_{j=0}^{N} D_{i j} \delta_{j k}+\widetilde{\nu} \partial_{x_{k}} e\left(x_{0}, x_{N}\right) \quad k=0, N \\
& =w_{k} \partial_{x_{k}} H\left(\widetilde{\lambda}_{k}, x_{k}, u_{k}\right)-\sum_{i=0}^{N} w_{i} \widetilde{\lambda}_{i} D_{i k}+\widetilde{\nu} \partial_{x_{k}} e\left(x_{0}, x_{N}\right)
\end{aligned}
$$

From Lemma 3, this implies,

$$
\begin{aligned}
& \partial_{x_{k}} \overline{J^{N}}[\widetilde{\lambda}, \widetilde{\nu}, X, U]=w_{k}\left(\partial_{x_{k}} H\left(\widetilde{\lambda}_{k}, x_{k}, u_{k}\right)+\sum_{i=0}^{N} D_{k i}^{*} \widetilde{\lambda}_{i}\right)+\widetilde{\lambda}_{k}^{*}+\widetilde{\nu} \partial_{x_{k}} e\left(x_{0}, x_{N}\right)=0, \quad k=0 \\
& \partial_{x_{k}} \overline{J^{N}}[\widetilde{\lambda}, \widetilde{\nu}, X, U]=w_{k}\left(\partial_{x_{k}} H\left(\widetilde{\lambda}_{k}, x_{k}, u_{k}\right)+\sum_{i=0}^{N} D_{k i}^{*} \widetilde{\lambda}_{i}\right)+\widetilde{\lambda}_{k}^{*}-\widetilde{\nu} \partial_{x_{k}} e\left(x_{0}, x_{N}\right)=0, \quad k=N
\end{aligned}
$$

where $\widetilde{\lambda}_{k}^{*}$ is given by:

\section{Legendre-Gauss}

$\tilde{\lambda}_{k}^{*}=0$ for $k=0$ and $N$

\section{Legendre-Gauss-Radau}

$\widetilde{\lambda}_{k}^{*}=\widetilde{\lambda}_{k}$ for $k=0$ and $\widetilde{\lambda}_{k}^{*}=0$ for $k=N$ 


\section{Legendre-Gauss-Lobatto}

$\widetilde{\lambda}_{k}^{*}=\widetilde{\lambda}_{k}$ for $k=0$ and $N$

Thus Problem $B^{N \lambda}$ can be written as,

$$
\left(B^{N \lambda}\right)\left\{\begin{array}{rlr}
\sum_{j=0}^{N} D_{i j} x_{j}-f\left(x_{i}, u_{i}\right) & =0 & i=0,1, \ldots, N \\
e\left(x_{0}, x_{N}\right) & =0 & \\
\sum_{j=0}^{N} D_{i j}^{*} \widetilde{\lambda}_{j}+\partial_{x_{i}} H\left(\widetilde{\lambda}_{i}, x_{i}, u_{i}\right) & =0 & i=1, \ldots, N-1 \\
\partial_{u_{i}} H\left(\widetilde{\lambda}_{i}, x_{i}, u_{i}\right) & =0 & i=0,1, \ldots, N \\
\sum_{j=0}^{N} D_{i j}^{*} \widetilde{\lambda}_{j}+\partial_{x_{i}} H\left(\widetilde{\lambda}_{i}, x_{i}, u_{i}\right) & =-c_{0}^{*} & i=0 \\
\sum_{j=0}^{N} D_{i j}^{*} \widetilde{\lambda}_{j}+\partial_{x_{i}} H\left(\widetilde{\lambda}_{i}, x_{i}, u_{i}\right) & =-c_{N}^{*} & i=N \\
\widetilde{\lambda}_{0}^{*}+\frac{\partial \bar{E}}{\partial x_{0}}\left(\widetilde{\nu}, x_{0}, x_{N}\right) & =w_{0} c_{0}^{*} \\
\widetilde{\lambda}_{N}^{*}-\frac{\partial \bar{E}}{\partial x_{N}}\left(\widetilde{\nu}, x_{0}, x_{N}\right) & =w_{N} c_{N}^{*}
\end{array}\right.
$$

where $c_{0}^{*}$ and $c_{N}^{*}$ are arbitrary real numbers.

The collection of problems developed in the preceding sections can be summarized by the commutative diagram promulgated in Ref. [41] and illustrated in Fig. 1. Now suppose that a solution to Problem $B^{\lambda N}$

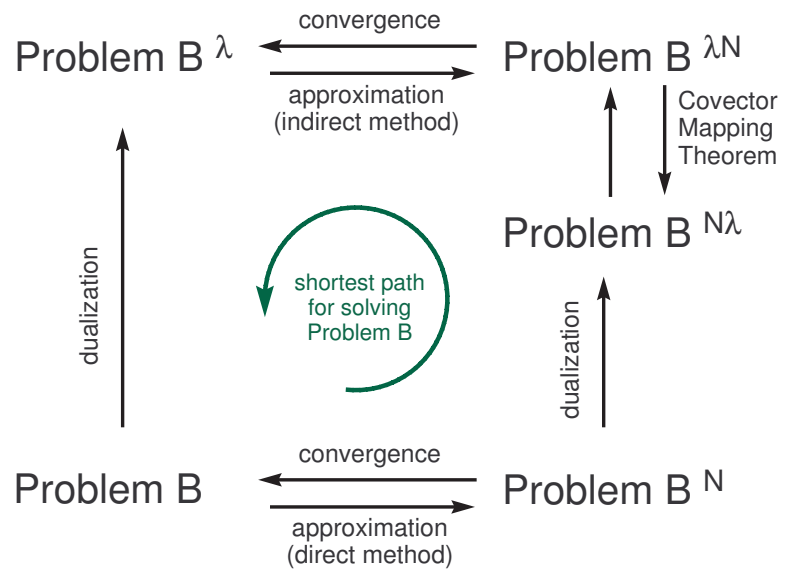

Figure 1. Development of the Covector Mapping Theorem.

exists. That is, we suppose that a (discretized) solution to the two-point BVP exists. Then the covectors of Problem $B^{\lambda N}$ provide an existence theorem for the solution of Problem $B^{N \lambda}$; that is, for all $k=0, \ldots, N$, $\widetilde{\lambda}_{k}=\lambda_{k}$ and $\widetilde{\nu}=\nu$ under the following conditions:

\section{Legendre-Gauss}

$x_{0}$ and $x_{N}$ are free; i.e. unspecified.

\section{Legendre-Gauss-Radau}

$x_{0}$ may be arbitrarily specified but $x_{N}$ must be free, or vice versa. 
Both $x_{0}$ and $x_{N}$ may be arbitrarily specified.

Theorem 1 (Covector Mapping Theorem) An existence of a solution to the two-point boundary value problem (Problem $B^{\lambda N}$ ) implies the existence of a solution to the KKT conditions (Problem $B^{N \lambda}$ ) for all Legendre PS methods under appropriate boundary conditions.

Remark 1 The main assumption of Theorem 1 is the existence of a solution to the two point BVP, Problem $B^{\lambda N}$. In practice, is it is customary to assume that a solution to the BVP exists. Consequently, Theorem 1 is a strong theorem under mild assumptions.

Remark 2 A simple counter example is constructed in Ref. [42] to show that a solution to Problem $B^{\lambda N}$ may not exist for Euler discretization no matter how small the mesh. This well-known phenomenon requires a proper technical modification to Theorem 1 similar to Polak's theory of consistent approximations. This aspect of Theorem 1 is rigorously proved in Refs. [46] and [48].

\section{A Minimalist's Approach to Solving Trajectory Optimization Problems}

The Covector Mapping Theorem (CMT) suggests an "embarrassingly simple" 55 approach to solving trajectory optimization problems. Under the validity of the CMT, there is no longer a need to develop all the necessary conditions for optimality and solve for these conditions. A substantially more robust and simple approach is to solve for the KKT conditions. The robustness of the approach comes from the fact that KKT system can be solved sequentially using augmented Lagrangian techniques that ensure global convergence under mild conditions. ${ }^{56}$ The simplicity of the approach comes from the fact that Problem $B^{N \lambda}$ can be automatically generated from a mere definition of Problem $B^{N}$. The CMT thus provides the critical missing link to ensure that the solution to Problem $B^{N \lambda}$ is the same as the solution to Problem $B^{\lambda N}$ under mild and checkable conditions; see Fig. 1. This is, in fact, the principle around which the software package, DIDO,$^{57}$ is constructed. That is, only the problem formulation, Problem $B$ is required as an "input" in much the same way as one writes the problem using pencil and paper. All of the necessary conditions are then automatically generated and checked. This does not imply that the PMP is no longer necessary to solve trajectory optimization problems. On the contrary, it is quite the opposite. The automated generation of states, controls, covectors and other dual maps provides a means - by way of the PMP — to eliminate spurious solutions. In many engineering applications, a check of a few necessary conditions (rather than all) are quite sufficient to include or exclude candidate solutions. Extensive examples of an application of the CMT are already noted in Section I.

\section{A Numerical Example}

To illustrate the ideas discussed so far, we consider the much-studied orbit transfer problem of Moyer and Pinkham ${ }^{58}$ that has evolved over the last forty years to a benchmark space trajectory optimization problem. See Ref. [18] for a brief history of this problem and some new benchmarks. The classical problem is formulated as finding the steering angle, $\beta(\tau)$ that maximizes the final radius at $\tau_{f}=3.32$. Therefore, the cost function is

$$
J[\mathbf{x}(\cdot), \mathbf{u}(\cdot)]=-r\left(\tau_{f}\right)
$$

where $\mathbf{x}^{T}=\left[r, \theta, v_{r}, v_{t}\right]$ and $\mathbf{u}=\beta$. The state variables are the radial distance $r$, the true anomaly $\theta$, the radial velocity component $v_{r}$, and the transverse velocity component $v_{t}$. The control variable is the thrust steering angle measured from the local horizontal $\beta$. The equations of motion are 


$$
\begin{aligned}
\frac{d r}{d \tau} & =v_{r} \\
\frac{d \theta}{d \tau} & =\frac{v_{t}}{r} \\
\frac{d v_{r}}{d \tau} & =\frac{v_{t}^{2}}{r}-\frac{1}{r^{2}}+A(\tau) \sin \beta \\
\frac{d v_{t}}{d \tau} & =-\frac{v_{r} v_{t}}{r}+A(\tau) \cos \beta
\end{aligned}
$$

where $A(\tau)$ is the control acceleration parameterized by

$$
A(\tau)=\frac{T}{\left(m_{0}-|\dot{m}| \tau\right)}
$$

where $m_{0}$ is the initial mass and $\dot{m}$ is the constant fuel consumption rate. The boundary conditions are

$$
\begin{array}{ll}
r(0)=1.0 & \theta(0)=0 \\
v_{r}(0)=0 & v_{t}(0)=1.0 \\
v_{r}\left(\tau_{f}\right)=0 & v_{t}\left(\tau_{f}\right)-\sqrt{1 / r\left(\tau_{f}\right)}=0
\end{array}
$$

The normalized constants for this problem are $m_{0}=1.0, T=0.1405, \tau_{f}=3.32$, and $|\dot{m}|=0.0749$.

We use this problem to investigate the effect of choice of the nodes on the solutions. Three separate cases of LGL, LGR and LG PS methods are considered. In all three cases we arbitrarily choose $N=32$. The solution for the states and controls for all three methods are shown in Figs. 2 and 3 respectively. In all the subsequent graphs, the solutions from the LGL method are denoted by $*$, the LG solutions are denoted by + and $o$ is used to denote the LGR solutions. It is apparent that all three methods show numerically

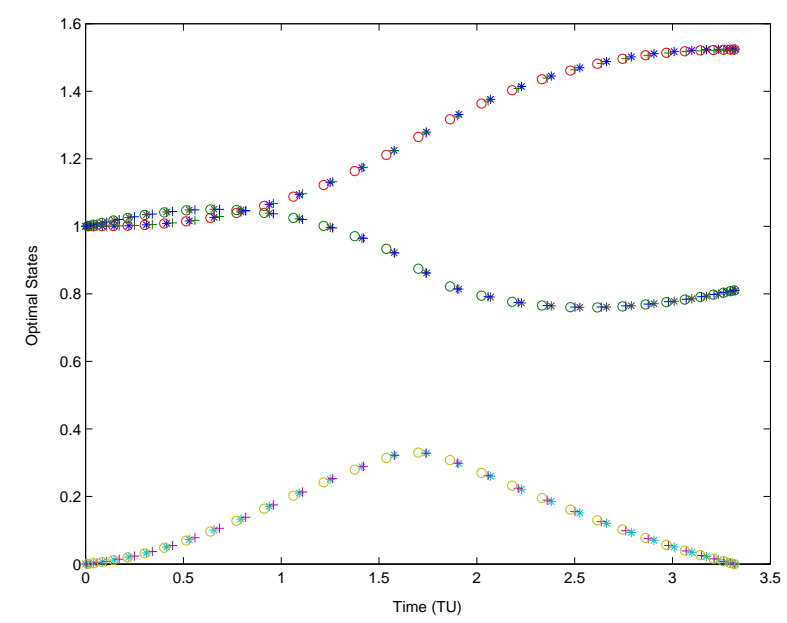

Figure 2. State trajectories for the LG, LGR and LGL pseudospectral methods.

indistinguishable performances despite that the LGL PS method is the only legitimate method to solve this problem since endpoint conditions are specified at both ends.

The mapped KKT multiplier trajectories based on Theorem 1 are shown in Fig. 4 for all three methods. Based on the extensive study of this problem, it is clear that these mapped KKT multipliers are indeed the costate trajectories.

In order to draw the final connections between theory and practice, we plot the unmapped KKT multipliers. Shown in Fig. 5 are the multiplier trajectories obtained when the wrong Hilbert space (i.e. unweighted) 


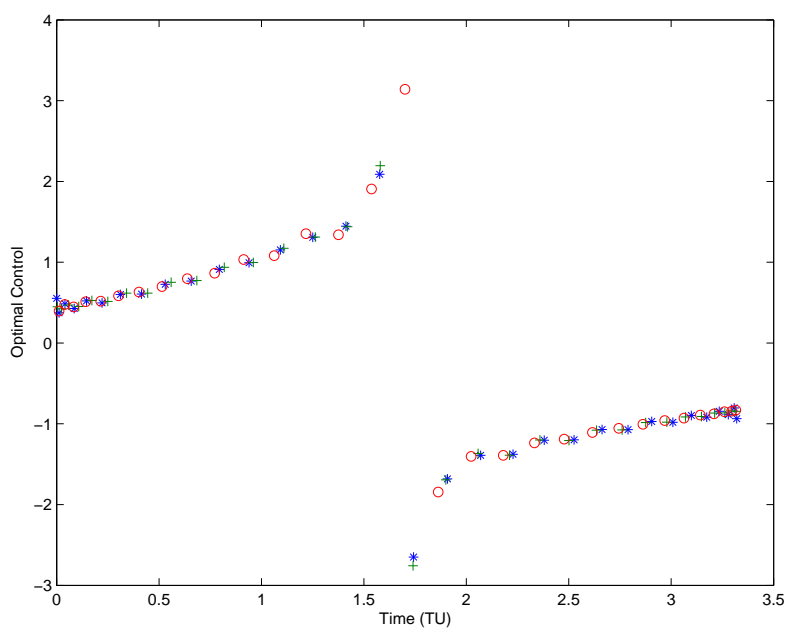

Figure 3. Control trajectories for the LG, LGR and LGL pseudospectral methods.

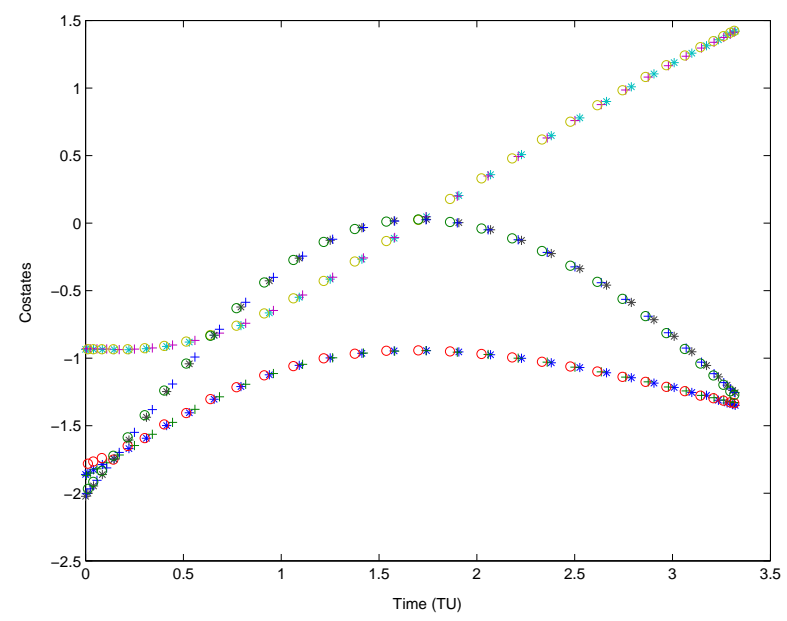

Figure 4. Mapped KKT multiplier trajectories for the LG, LGR and LGL pseudospectral methods.

is used to imply a connection between the KKT multipliers and the costate trajectories. Clearly, none of the KKT multipliers can be construed as discrete costate trajectories. Next, the multiplier trajectories from the correct Hilbert space but a wrong mapping is plotted in Fig. 6. It is not surprising that a proper use of the theory generates the right answer!

As a final point of contention we note that it is incorrect to interpret the KKT multiplier trajectories shown in Fig. 6 as "wiggles" about the correct answer. The appearance of the wiggles is simply a result of existence of a primal solution in the absence of satisfying the KKT constraint qualifications. A proof of this assertion is beyond the scope of this paper but may be found in Refs. $[24,34,35,42,45,47,48,59,60]$ with various levels of sophistication and rigor.

\section{Some Remarks on the Completeness of Our Framework}

In order to support a wide exposition of PS methods for optimal control, we have taken the liberty of describing our core ideas in terms of certain simplifications. As we briefly noted in Sec. II, this is not to be confused as a limitation of the ideas; rather, with additional bookkeeping all our ideas transfer 


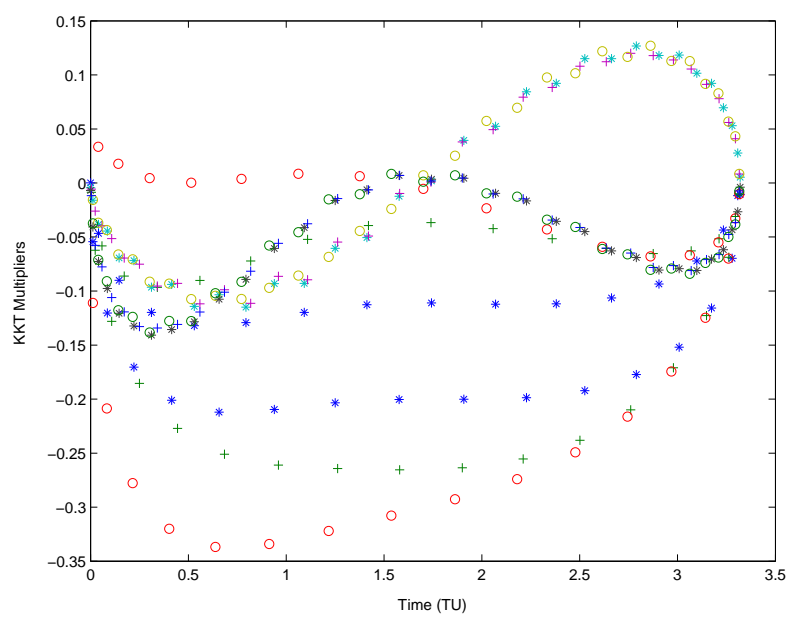

Figure 5. KKT multiplier trajectories in the wrong Hilbert space.

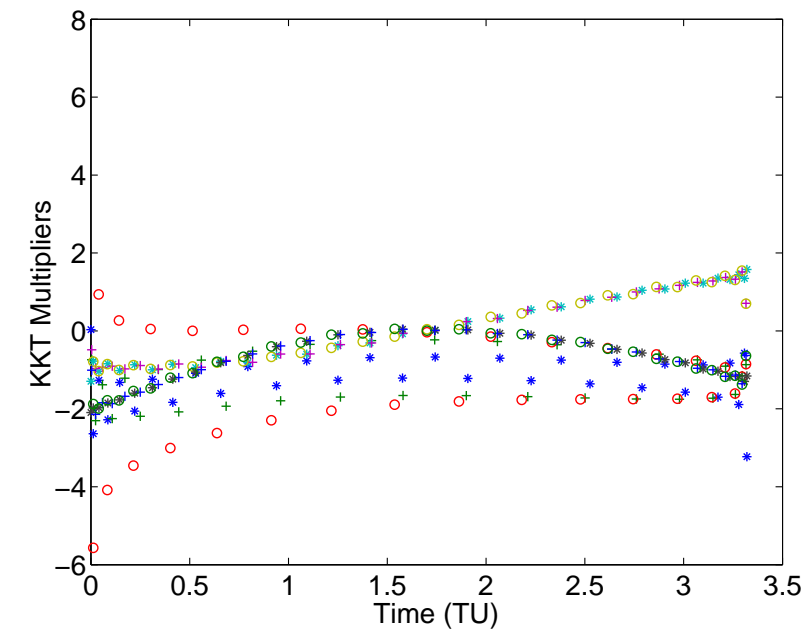

Figure 6. KKT multiplier trajectories in the correct Hilbert space but wrong mapping.

trivially to substantially more complex problems than the one posed as Problem $B$. In the same spirit, we have restricted the discussions in this paper to Legendre-based PS methods. Our ideas apply equally to other PS methods as well. The key new concept we have proposed in this paper is contained in Eq. (14). This is the notion of consistent primal and dual weight functions for interpolation. Thus PS methods for optimal control are now firmly established as being distinct from PS methods in other applications. Roughly speaking, PS methods for optimal control can be described as follows: For any chosen primal interpolant with primal weight function, $W(t)$, it is necessary to choose a consistent dual interpolant with weight function, $W^{*}(t)$. The weight function pair, $\left\{W(t), W^{*}(t)\right\}$, must be selected in a manner that generates the correct Hilbert space for the approximation of functions. Under the assumption of existence of a continuous-time solution in an appropriate Sobolev space, global convergence of the solution based on the consistency of the approximation can then be rigorously proved under mild and checkable conditions. Refs. [46, 48] present some of the foundations for such results. These theoretical developments explain the consistently superior performance of PS methods for optimal control. A generalized presentation of these ideas is beyond the scope of this paper, but it is now clear why the Lobatto family of Legendre PS methods have a weight function of unity. For non-unit weight functions, the starting point for designing PS methods is Eq. (14). 


\section{Conclusions}

Since the early days of the calculus of variations it was well-recognized that nowhere was it more probable than this field to arrive at the right answer by a wrong approach. Optimal control theory is filled with many subtleties and nuances. A proper application of discrete methods requires one to be fully cognizant of these apparently minor technicalities. Based on the authors' work on PS methods and Hager's on RK methods, it has become clear in recent years that dual consistency is not a mere luxury to solve optimal control problems; rather, it is quite essential for a correct solution to the problem. When the problem is correctly discretized, the Covector Mapping Principle (CMP) provides a simple and elegant escape from the trials and tribulations of solving two-point boundary value problems. In other words, the CMP implies that a vast number of space trajectory optimization problems should no longer be considered as difficult problems. This does not imply the end of research in trajectory optimization techniques; rather, an exciting new beginning where theory and practice are juxtaposed.

\section{Acknowledgments}

We gratefully acknowledge partial funding for this research provided to one of the authors (Ross) by the Air Force Office of Scientific Research under AFOSR Grant F1ATA0-60-6-2G002.

\section{References}

\footnotetext{
${ }^{1}$ Stevens, R., Ross, I. M., and Matousek, S. E., "Earth-Mars Return Trajectories Using Solar Sails," 55th International Astronautical Congress, Vancouver, Canada. Paper IAC-04-A.2.08, October 4-8, 2004.

${ }^{2}$ Hawkins, A. M., Fill, T. R., Proulx, R. J., Feron, E. M., "Constrained Trajectory Optimization for Lunar Landing," $A A S$ Spaceflight Mechanics Meeting, Tampa, FL, January 2006, AAS 06-153.

${ }^{3}$ Infeld, S. I. and Murray, W., "Optimization of Stationkeeping for a Libration Point Mission," AAS Spaceflight Mechanics Meeting, Maui, HI, February 2004. AAS 04-150.

${ }^{4}$ Stevens, R. and Ross, I. M., "Preliminary Design of Earth-Mars Cyclers Using Solar Sails," Journal of Spacecraft and Rockets, Vol. 41, No. 4, 2004.

${ }^{5}$ Ross, I. M. and D'Souza, C. D., "Hybrid Optimal Control Framework for Mission Planning," Journal of Guidance, Control and Dynamics, Vol. 28, No. 4, July-August 2005, pp. 686-697.

${ }^{6}$ Croley, P. A., "Reachable Sets for Multiple Asteroid Sample Return Missions," Astronautical Engineer's Degree Thesis, Naval Postgraduate School, Monterey, CA, December 2005.

${ }^{7} \mathrm{P}$. Williams, Application of Pseudospectral Methods for Receding Horizon Control, J. of Guid., Contr. and Dyn., Vol.27, No.2., 2004, pp.310-314.

${ }^{8} \mathrm{P}$. Williams, C. Blanksby and P. Trivailo, Receding horizon control of tether system using quasilinearization and Chebyshev pseudospectral approximations, AAS/AIAA Astrodynamics Specialist Conference, Big Sky, MT, August 3-7, 2003, Paper AAS 03-535.

${ }^{9}$ Infeld, S. I., Josselyn, S. B., Murray W. and Ross, I. M., "Design and Control of Libration Point Spacecraft Formations," Journal of Guidance, Control and Dynamics, to appear.

${ }^{10}$ Josselyn, S. B. and Ross, I. M., "Optimization of Low Thrust Trajectories with Terminal Aerocapture Maneuvers," 55th International Astronautical Congress, Vancouver, Canada, IAC-04-IAA.6.05, October 4-8, 2004.

${ }^{11}$ Rea, J., "Launch Vehicle Trajectory Optimization Using a Legendre Pseudospectral Method," Proceedings of the AIAA Guidance, Navigation and Control Conference, Austin, TX, August 2003. Paper No. AIAA 2003-5640.

${ }^{12}$ P. Lu, H. Sun and B. Tsai, Closed-Loop Endoatmospheric Ascent Guidance, Journal of Guidance, Control and Dynamics, Vol. 26, No. 2, pp.283-294, 2003.

${ }^{13}$ Josselyn S. and Ross, I. M., "A Rapid Verification Method for the Trajectory Optimization of Reentry Vehicles," Journal of Guidance, Control and Dynamics, Vol. 26, No. 3, 2003.

${ }^{14}$ Fahroo, F., Doman, D. and Ngo, A., "Modeling Issues in Footprint Generation for Reusable Launch Vehicles," Proceedings of the 2003 IEEE Aerospace Conference, Big Sky, MT, March 2003.

${ }^{15}$ Fahroo, F., Doman, D. and Ngo, A., "Footprint Generation for Reusable Launch Vehicles Using a Direct Pseudospectral Method," Proceedings of the American Control Conference, Denver, CO June 2003.

${ }^{16}$ Fahroo, F. and Doman, D., "A Direct Method for Approach and Landing Trajectory Reshaping with Failure Effect Estimation," 2004 AIAA Conference on Guidance, Navigation and Control, August 2004, Providence, RI.

${ }^{17}$ Ross, I. M., King, J. T., Fahroo, F., "Designing Optimal Spacecraft Formations," Proceedings of the AIAA/AAS Astrodynamics Conference, AIAA-2002-4635, Monterey, CA, 5-8 August 2002.

${ }^{18}$ Ross, I. M., "Space Trajectory Optimization and $L^{1}$-Optimal Control Problems," Modern Astrodynamics, Elsevier, 2006, Chapter 6 .

${ }^{19}$ Mendy, P. B., "Multiple Satellite Trajectory Optimization," Astronautical Engineer Thesis, Department of Mechanical and Astronautical Engineering, Naval Postgraduate School, Monterey, CA, December 2004.

${ }^{20}$ Ross, I. M., Gong, Q. and Sekhavat, P., "A Simple Technique for Low-Thrust High-Accuracy Trajectory Optimization," 16th AAS/AIAA Space Flight Mechanics Conference, Tampa, FL, January 22-26, 2006. AAS 06-150.
} 
${ }^{21}$ Fahroo, F. and Ross, I. M., "Costate Estimation by a Legendre Pseudospectral Method," Journal of Guidance, Control and Dynamics, Vol.24, No.2, March-April 2001, pp.270-277.

${ }^{22}$ Elnagar, J., Kazemi, M. A. and Razzaghi, M., "The Pseudospectral Legendre Method for Discretizing Optimal Control Problems," IEEE Transactions on Automatic Control, Vol. 40, No. 10, 1995, pp. 1793-1796.

${ }^{23} \mathrm{~S}$. Stanton, R. Proulx and C. D'Souza, Optimal orbit transfer using a Legendre pseudospectral method, AAS/AIAA Astrodynamics Specialist Conference, AAS-03-574, Big Sky, MT, August 3-7, 2003.

${ }^{24}$ Ross, I. M., "A Roadmap for Optimal Control: The Right Way to Commute," Annals of the New York Academy of Sciences, Vol. 1065, January 2006.

${ }^{25}$ Fleming, A. and Ross, I. M., "Singularity-Free Optimal Steering of Control Moment Gyros," Proceedings of the 2005 AAS/AIAA Astrodynamics Specialist Conference, August 7-11, 2005 Lake Tahoe, CA. AAS 05-418.

${ }^{26} \mathrm{~J}$. Pietz and N. Bedrossian, "Moemtum Dumping Using Only CMGs," Proceedings of the AIAA GNC Conference, Austin, TX, 2003.

${ }^{27}$ H. Yan and K. T. Alfriend, Three-axis Magnetic Attitude Control Using Pseudospectral Control Law in Eccentric Orbits, AAS Spaceflight Mechanics Meeting, Tampa, FL, January 2006, AAS 06-103.

${ }^{28}$ Sekhavat, P., Fleming A. and Ross, I. M., "Time-Optimal Nonlinear Feedback Control for the NPSAT1 Spacecraft," Proceedings of the 2005 IEEE/ASME International Conference on Advanced Intelligent Mechatronics, AIM 2005, 2428 July 2005 Monterey, CA.

${ }^{29}$ Harada, M., Bollino, K., and Ross, I. M., "Minimum-Fuel Circling for an Unmanned Aerial Vehicle," 2005 JSASS-KSAS Joint International Symposium on Aerospace Engineering, Nagoya, Japan, October 12-15, 2005.

${ }^{30}$ I. M. Ross and F. Fahroo, Pseudospectral methods for optimal motion planning of differentially flat systems, IEEE Trans. on Automat. Contr., Vol. 49, No. 8, pp. 1410-1413, August 2004.

${ }^{31}$ Ross, I. M. and Fahroo, F., "Issues in the Real-Time Computation of Optimal Control," Mathematical and Computer Modelling, Vol. 40, Pergamon Publication (to appear).

${ }^{32}$ Gong, Q., Ross, I. M. and Kang, W., "A Pseudospectral Observer for Nonlinear Systems," Proceedings of the AIAA Guidance, Navigation and Control Conference, August 2005, San Franscisco, CA.

${ }^{33}$ Paris, S. W. and Hargraves, C. R., OTIS 3.0 Manual, Boeing Space and Defense Group, Seattle, WA, 1996.

${ }^{34}$ Ross, I. M. and Fahroo, F., "A Pseudospectral Transformation of the Covectors of Optimal Control Systems," Proceedings of the First IFAC Symposium on System Structure and Control, Prague, Czech Republic, 29-31 August 2001.

${ }^{35}$ Ross, I. M. and Fahroo, F., "Legendre Pseudospectral Approximations of Optimal Control Problems," Lecture Notes in Control and Information Sciences, Vol.295, Springer-Verlag, New York, 2003.

${ }^{36}$ J. T. Betts, "Survey of Numerical Methods for Trajectory Optimization" Journal of Guidance, Control, and Dynamics, Vol 21, No. 2, 1998, 193-207.

${ }^{37}$ J. T. Betts, Practical Methods for Optimal Control Using Nonlinear Programming, SIAM, Philadelphia, PA, 2001.

${ }^{38}$ Hager, W. W., "Numerical Analysis in Optimal Control," International Series of Numererical Mathematics, Hoffmann, K.-H. Lasiecka, I., Leugering, G., Sprekels, J., and Troeltzsch, F., Eds., Birkhäuser, Basel, Switzerland, 2001, Vol. 139, pp. 83-93.

${ }^{39}$ Mordukhovich, B. S., Variational Analysis and Generalized Differentiation, I: Basic Theory, vol. 330 of Grundlehren der Mathematischen Wissenschaften [Fundamental Principles of Mathematical Sciences] Series, Springer, Berlin, 2005.

${ }^{40}$ Mordukhovich, B. S., Variational Analysis and Generalized Differentiation, II: Applications, vol. 331 of Grundlehren der Mathematischen Wissenschaften [Fundamental Principles of Mathematical Sciences] Series, Springer, Berlin, 2005.

${ }^{41}$ Ross, I. M. and Fahroo, F., "A Perspective on Methods for Trajectory Optimization," Proceedings of the AIAA/AAS Astrodynamics Conference, Monterey, CA, August 2002. AIAA Paper No. 2002-4727.

${ }^{42}$ Ross, I. M., "A Historical Introduction to the Covector Mapping Principle," AAS/AIAA Astrodynamics Specialist Conference, Tahoe, NV, August 8-11, 2005, Paper AAS 05-332.

${ }^{43}$ Fahroo, F. and Ross, I. M., "Pseudospectral Methods for Infinite Horizon Nonlinear Optimal Control Problems," AIAA Guidance, Navigation and Control Conference, San Francisco, CA, 2005.

${ }^{44}$ Ross, I. M., Gong, Q., Fahroo, F. and Kang, W., "Practical Stabilization Through Real-Time Optimal Control," 2006 American Control Conference, Minneapolis, MN, June 14-16 2006.

${ }^{45}$ Ross, I. M. and Fahroo, F., "Discrete Verification of Necessary Conditions for Switched Nonlinear Optimal Control Systems," Proceedings of the American Control Conference, June 2004, Boston, MA.

${ }^{46}$ Gong, Q., Kang, W. and Ross, I. M., "A Pseudospectral Method for the Optimal Control of Constrained Feedback Linearizable Systems," IEEE Transactions on Automatic Control, Vol. 51, No. 7, July 2006, pp. 1115-1129.

${ }^{47}$ Gong, Q., Ross, I. M., Kang, W. and Fahroo, F., "Dual Convergence of the Legendre Pseudospectral Method for Solving Nonlinear Constrained Optimal Control Problems," Proceedings of the IASTED International Conference on Intelligent Systems and Control, Cambridge, MA, 2005.

${ }^{48}$ Gong, Q., Ross, I. M., Kang, W. and Fahroo, F., "On the Pseudospectral Covector Mapping Theorem for Nonlinear Optimal Control," to appear in Proceedings of the 45th IEEE Conference on Decision and Control, San Diego, CA, December 13-15, 2006.

${ }^{49}$ Vinter, R. B., Optimal Control, Birkhäuser, Boston, MA, 2000.

${ }^{50}$ Canuto, C., Hussaini, M. Y., Quarteroni, A., and Zang, T. A., Spectral Methods in Fluid Dynamics, Springer Verlag, New York, 1988.

${ }^{51}$ Weideman, J.A.C., "Spectral Methods Based on Non-Classical Orthogonal Polynomials," The Proceedings of the Oberwolfach Meeting on Application and Computation of Orthogonal Polynomials, March 1998.

${ }^{52}$ Fahroo, F. and Ross, I. M., "Direct Trajectory Optimization by a Chebyshev Pseudospectral Method," Journal of Guidance, Control and Dynamics, Vol. 25, No. 1, 2002, pp.160-166.

${ }^{53}$ Davis, P., and Rabinowitz, P., Methods of Numerical Integraion, Academic Press, 1975. 
${ }^{54}$ Karniadakis, G., and Sherwin, S., Spectal/hp Element Methods for Computational Fluid Dynamics, Oxford University Press, Oxford, 2005.

${ }^{55}$ Trefethen, L. N., Spectral Methods in MATLAB, SIAM, Philadelphia, PA, 2000.

${ }^{56}$ Gill, P. E., Murray, W. and Saunders, M. A., "SNOPT: An SQP Algorithm for Large-Scale Constrained Optimization," SIAM Review, Vol. 47, No. 1, 2005, pp. 99-131.

${ }^{57}$ Ross, I. M., User's Manual for DIDO: A MATLAB Application Package for Solving Optimal Control Problems, Technical Report 04-01.0, Tomlab Optimization Inc, February 2004.

${ }^{58}$ Moyer, H. G., and Pinkham, G., "Several Trajectory Optimization Techniques, Part II: Applications," Computing Methods in Optimization Problems, edited by A. V. Balakrishnan, and L. W. Neustadt, New York, Academic Press, 1964, pp. 91-105.

${ }^{59}$ Ross, I. M., "Certain Connections in Optimal Control Theory and Computation," Proceedings of the Symposium on New Trends in Nonlinear Dynamics and Control and Their Applications, Invited Talk, 18-19 October 2002, Monterey, CA.

${ }^{60}$ Ross, I. M., "A Framework for Analyzing Discrete Approximations in Optimal Control," SIAM Conference on Mathematics for Industry: Challenges and Frontiers, Invited Talk, Toronto, Ontario, Canada, 13-15 October 2003. 